\title{
Anti-prolactin (PRL) autoantibody-binding sites (epitopes) on PRL molecule in macroprolactinemia
}

\author{
N Hattori, Y Nakayama, K Kitagawa, $\mathbf{T}$ Ishihara ${ }^{1}$, Y Saiki ${ }^{1}$ and C Inagaki \\ Department of Pharmacology, Kansai Medical University, 10-15 Fumizono-cho, Moriguchi-City, Osaka 570-8506, Japan \\ ${ }^{1}$ Department of Endocrinology, Kobe City General Hospital, Kobe 650-0046, Japan \\ (Requests for offprints should be addressed to N Hattori; Email: hattorin@takii.kmu.ac.jp)
}

\begin{abstract}
Macroprolactinemia, in which serum prolactin (PRL) mainly consists of PRL with a molecular mass greater than $100 \mathrm{kDa}$, has been demonstrated to be associated with hyperprolactinemia. We previously reported that anti-PRL autoantibody is the major cause of macroprolactinemia. In this study, the autoantibodybinding sites (epitopes) on the PRL molecule were examined using deletion mutant PRL. The sera from 159 patients with hyperprolactinemia were screened for macroprolactinemia using the polyethylene glycol method and 18 patients (11\%) were diagnosed with macroprolactinemia. The sera from these patients were incubated with glutathione S-transferase-human prolactin (hPRL) fragment fusion proteins immobilized on glutathione sepharose and the amounts of bound
\end{abstract}

immunoglobulin $\mathrm{G}(\mathrm{IgG})$ were measured using ELISA. IgG was bound to full-length hPRL1-199 in significantly greater amounts in sera from 14 of 18 patients with macroprolactinemia than in controls. hPRL, but not PRL of other species such as bovine, porcine, rat, or human $\mathrm{GH}$, dose-dependently displaced the binding, suggesting that these patients had hPRL-specific autoantibodies. Deletion of 34 amino acid residues from Nand/or C-terminals significantly reduced the binding and $\mathrm{N}$ - or C-terminal fragment alone showed partial but significant binding, suggesting that the major epitopes recognized by anti-PRL autoantibodies are located in both $\mathrm{N}$ - and C-terminal residues of the PRL molecule.

Journal of Endocrinology (2006) 190, 287-293

\section{Introduction}

Recently, growing evidence has suggested that macroprolactinemia is associated with hyperprolactinemia (Leslie et al. 2001, Vallette-Kasic et al. 2002, Gibney et al. 2005). Macroprolactinemia is a condition of hyperprolactinemia in which serum prolactin (PRL) consists mainly of big-big PRL (macroprolactin) with a molecular mass greater than $100 \mathrm{kDa}$, in contrast to the predominant 23-kDa monomeric PRL as seen in other hyperprolactinemic states (Sinha 1995). The clinical symptoms of hyperprolactinemia such as amenorrhea and galactorrhea are largely missing and spontaneous pregnancy is possible without medication (Whittaker et al. 1981). Several studies have revealed that the prevalence of macroprolactinemia is unexpectedly high, $10-46 \%$ among patients with hyperprolactinemia. Since the prevalence of idiopathic hyperprolactinemia has been reported to be $8 \cdot 5-$ $40 \%$ of the whole hyperprolactinemia (Kleinberg et al. 1977, Sluijmer \& Lappöhn 1992), many of those who have been diagnosed with idiopathic hyperprolactinemia are possibly included in this new category.

Despite many reports about the prevalence, clinical manifestations, and laboratory diagnosis of macroprolactinemia, little is known about the formation of macroprolactin. Several groups and ourselves have identified anti-PRL autoantibodies in the sera from patients with macroprolactinemia (Hattori et al. 1992, Cavaco et al. 1995, Pascoe-Lira et al. 2001, De Schepper et al. 2003), though much remains unknown about their biological significance. In this study, we examined autoantibody-binding sites (epitopes) on the PRL molecule using PRLdeletion mutants, and compared them with the binding sites to PRL receptors to clarify the effects of the autoantibodies on the PRL bioactivity.

\section{Materials and Methods}

\section{Subjects}

We screened macroprolactinemia in 159 hyperprolactinemic patients (12 males and 147 females, aged 13-69 years), including 76 with prolactinoma, 7 with brain tumor, 28 drug-induced, and 48 idiopathic, using the polyethylene glycol (PEG) method (Hattori et al. 1992). Eighteen patients were diagnosed as having macroprolactinemia according to the criteria based on poor recovery of PRL after $12 \cdot 5 \%$ PEG serum treatment (recovery less than $40 \%$ of the total PRL).

The clinical characteristics of the 18 patients with macroprolactinemia are shown in Table 1. The initial PRL levels were measured for various reasons, including headache, abortion, abnormal genital bleeding, minimal galactorrhea, 
Table 1 The clinical characteristics of patients with macroprolactinemia

\begin{tabular}{|c|c|c|c|c|c|c|c|c|}
\hline & $\begin{array}{l}\text { Age } \\
\text { (years) }\end{array}$ & Sex & $\begin{array}{l}\text { Causes of } \\
\text { hyperprolactinemia }\end{array}$ & Menstruation & Galactorrhea & $\begin{array}{l}\text { Total PRL } \\
(\mu \mathrm{g} / \mathrm{L})\end{array}$ & $\begin{array}{l}\text { Free PRL }{ }^{1} \\
(\mu \mathrm{g} / \mathrm{L})\end{array}$ & $\begin{array}{l}\text { PEG }^{2} \\
(\%)\end{array}$ \\
\hline \multicolumn{9}{|l|}{ Patients } \\
\hline 1 & 41 & $\mathrm{~F}$ & Idiopathic & Regular & - & 225 & 6 & 3 \\
\hline 2 & 27 & $\mathrm{~F}$ & Idiopathic & Regular & - & 165 & 24 & 15 \\
\hline 3 & 67 & $\mathrm{~F}$ & Idiopathic & Postmenopause & - & 110 & 1 & 1 \\
\hline 4 & 41 & $\mathrm{~F}$ & Idiopathic & Oligomenorrhea & Minimal & 250 & 34 & 14 \\
\hline 7 & 55 & $\mathrm{~F}$ & Idiopathic & Postmenopause & - & 80 & 9 & 11 \\
\hline 8 & 39 & $\mathrm{~F}$ & Drug-induced & Regular & n.d. ${ }^{3}$ & 268 & 60 & 22 \\
\hline 9 & 37 & $\mathrm{~F}$ & Idiopathic & Regular & - & 54 & 21 & 39 \\
\hline 10 & 39 & $\mathrm{~F}$ & Idiopathic & Regular & - & 111 & 37 & 33 \\
\hline 11 & 24 & $\mathrm{~F}$ & Idiopathic & Regular & - & 51 & 18 & 35 \\
\hline 12 & 24 & $\mathrm{~F}$ & Idiopathic & Regular & - & 80 & 13 & 16 \\
\hline \multicolumn{9}{|l|}{$\mathbf{A b}(-)$} \\
\hline 15 & 40 & $\mathrm{~F}$ & Idiopathic & Regular & - & 33 & 4 & 12 \\
\hline 16 & 28 & M & Idiopathic & & - & 41 & 9 & 22 \\
\hline 17 & 38 & $\mathrm{~F}$ & Prolactinoma ${ }^{4}$ & Regular & - & 44 & 16 & 36 \\
\hline \multirow[t]{2}{*}{18} & 42 & M & Drug-induced & & $-^{5}$ & 40 & 7 & 18 \\
\hline & $38 \pm 9$ & & & & & $103 \pm 15$ & $16 \pm 3$ & $18 \pm 2$ \\
\hline Control $(n=23)$ & $36 \pm 2$ & & & & & $131 \pm 18$ & $129 \pm 17$ & $99 \pm 1$ \\
\hline
\end{tabular}

${ }^{1} \mathrm{PRL}$ concentration in the supernatant after $12 \cdot 5 \%$ PEG treatment.

${ }^{2}$ Free PRL/total PRL x $100 \%$

${ }^{3}$ n.d., not determined (information about galactorrhea was not available on her medical record).

${ }^{4}$ This patient was diagnosed as having prolactinoma by high serum PRL levels (450 $\left.\mu \mathrm{g} / \mathrm{l}\right)$ and MRI. She has been asymptomatic on dopaminergic agonist (terguride).

${ }^{5}$ Total and free testosterone concentrations were $3 \cdot 6 \mathrm{ng} / \mathrm{ml}$ (normal $2 \cdot 7-10 \cdot 7 \mathrm{ng} / \mathrm{ml}$ ) and $13 \cdot 5 \mathrm{pg} / \mathrm{ml}$ (normal $5 \cdot 1-19 \cdot 0 \mathrm{pg} / \mathrm{ml}$ ), respectively in patient 18 .

Testosterone concentrations were measured by RIA kit (Japan DPC Corp., Tokyo).

gynecomastia, anti-depressive drugs, and general endocrine evaluation. Age- and total PRL-matched 23 control patients were chosen from the 141 hyperprolactinemic patients without macroprolactinemia. Free PRL concentrations after treatment with PEG were all within normal limits $(\mathrm{PRL}<$ $27 \mu \mathrm{g} / 1$, mean $+2 \cdot 5$ s.D. in 42 normal women) except for three (cases 4, 8, and 10). The apparent causes for hyperprolactinemia were unknown in 15 of the 18 macroprolactinemic patients (idiopathic hyperprolactinemia) and they almost entirely lacked the clinical symptoms of hyperprolactinemia, such as amenorrhea and galactorrhea. Human, porcine, bovine, and rat PRL and human growth hormone $(\mathrm{GH})$ were kindly supplied by the National Hormone and Pituitary Program. This study was approved by the Ethical Committee of Kobe City General Hospital.

\section{Production of recombinant human prolactin (hPRL) fragments}

Total RNA was extracted from a human leukemic cell line (Jurkat) using RNeasy kit (Qiagen). The primers for hPRL were designed according to the published sequence of hPRL (NCBI accession number: NM_000948) 5'-AACATGAACATCAAAGGATCG-3' (forward) and 5'-TTAGCAGTTG TTGTTGTGGAT-3' (reverse) flanking full-length coding region of hPRL mRNA. Reverse transcriptase (RT)-PCR was performed with a commercially available RT-PCR kit (Invitrogen) under the following conditions: $50{ }^{\circ} \mathrm{C}$ for $30 \mathrm{~min}$ for cDNA synthesis followed by 40 cycles of PCR (denaturing at $94{ }^{\circ} \mathrm{C}$ for $15 \mathrm{~s}$, annealing at $54{ }^{\circ} \mathrm{C}$ for $30 \mathrm{~s}$ and extension at $72^{\circ} \mathrm{C}$ for $1 \mathrm{~min}$ ) in a GeneAmp PCR System 2400 (Perkin-Elmer Corp., Foster City, CA, USA). The PCR product was electrophoresed in 1\% agarose gel, purified using Gene Clean spin (BIO 101, Vista, CA, USA), ligated to a plasmid vector $(\mathrm{pCR} 2 \cdot 1)$ and amplified using a TA cloning kit (Invitrogen). The plasmid DNA was purified by an extraction kit (Toyobo, Osaka, Japan) and the sequence was confirmed to match hPRL cDNA by DNA-sequencing PCR using a Big Dye Termination Cycle Sequencing kit (Perkin-Elmer Corp.) in a DNA sequencer (Applied Biosystems, Foster City, CA, USA).

The cDNAs of hPRL fragments were produced by PCR using full-length hPRL cDNA as a template and specific primers including restriction enzyme sites (BamHI for forward and XhoI for reverse primers; Table 2). The PCR products were doubly digested with $\mathrm{BamHI}-\mathrm{XhoI}$ restriction endonucleases and ligated to plasmid pGEX 4T-1 (GE Healthcare Bio-Sciences Corp., Piscataway, NJ, USA), which had been digested similarly. The cDNAs of hPRL 
Table 2 The positions of primers

\section{Forward primer}

\section{PRL fragment}

PRL1-199

PRL35-199

PRL1-165

PRL35-165

PRL1-81

PRL135-199 $5^{\prime}$-ctaggatccttgcccatctgtcccgg-3'

$5^{\prime}$-ctaggatccgaaatgttcagcgaattc- $3^{\prime}$

$5^{\prime}$-ctaggatccttgcccatctgtcccgg- $3^{\prime}$

$5^{\prime}$-ctaggatccgaaatgttcagcgaattc- $3^{\prime}$

$5^{\prime}$-ctaggatccttgcccatctgtcccgg-3'

$5^{\prime}$-ctaggatcccaggttcatcctgaaac- $3^{\prime}$

\section{Reverse primer}

5'-agactcgagttagcagttgttgttgtgga-3'

$5^{\prime}$-agactcgagttagcagttgttgttgtgga- $3^{\prime}$

$5^{\prime}$-agactcgagttaaaggcgagactcttcat- $3^{\prime}$

$5^{\prime}$-agactcgagttaaaggcgagactcttcat- $3^{\prime}$

$5^{\prime}$-agactcgagttacagaaagtcttttgattc- $3^{\prime}$

$5^{\prime}$-agactcgagttagcagttgttgttgtgga-3'

Forward and reverse primers include BamHI (ggatcc) and Xhol (ctcgag) sites besides the coding regions, respectively.

and its 5 fragments inserted at the $3^{\prime}$-end of the glutathione S-transferase (GST) gene are schematically represented in Fig. 1a. Escherichia coli (DH5- $\alpha$ ) was transfected with the plasmids, grown in Lauria Bertani medium containing $100 \mu \mathrm{g} / \mathrm{ml}$ ampicillin, and the fusion protein expression was induced for $2 \mathrm{~h}$ at $37^{\circ} \mathrm{C}$ with $0.2 \mathrm{mmol} / 1$ isopropyl $\beta$-D-thiogalactoside. The fusion proteins were purified from bacterial lysates using Glutathione Sepharose 4B (GS4B) and analyzed by SDS-PAGE stained with Sypro Ruby (Molecular Probe, Eugene, OR, USA). The bands with estimated molecular masses and probably those derived from the partial degradation products were recognized (Fig. 1b).

(a)

\begin{tabular}{llll|} 
hPRL1-199 \\
hPRL35-199 \\
hPRL35-165
\end{tabular}

(b)

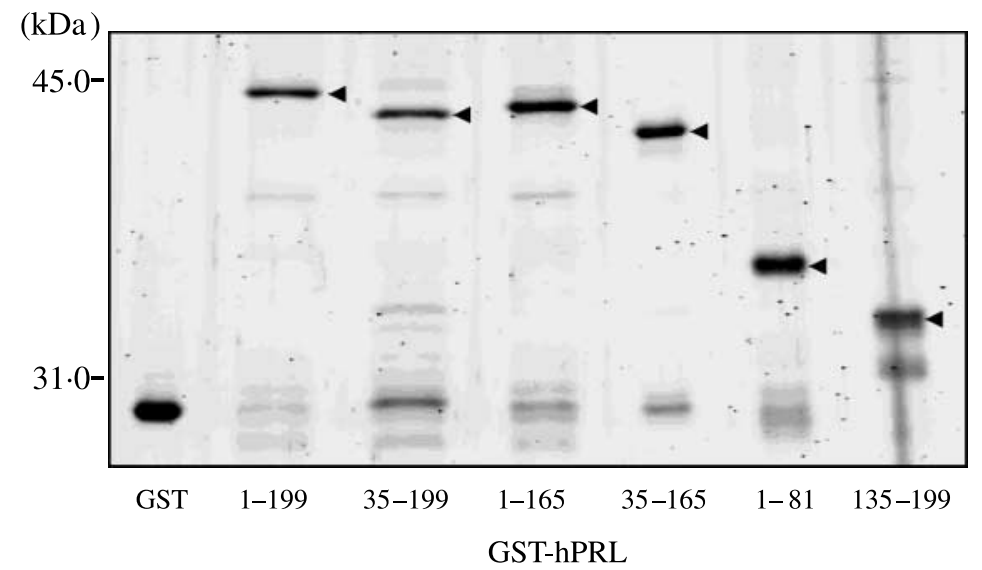

Figure 1 The positions of the hPRL-deleted mutants and SDS-PAGE analysis of the fusion proteins. (a) Full-length hPRL1-199 and deleted mutants were produced as GST fusion proteins in Escherichia coli using a pGEX system as described in Materials and Methods. (b) GST fusion proteins as well as GST alone were run on 10\% SDS-PAGE and stained with Sypro Ruby. The bands were visualized using an optical scanner (Fluorlmager 595). The arrow indicates the band of each fusion protein. 


\section{Production of solid phase}

The GS4B-bound fusion proteins (solid phase) were placed in $0 \cdot 01 \mathrm{~mol} / 1$ phosphate buffer, $\mathrm{pH} 7 \cdot 0$, containing $0 \cdot 2 \%$ BSA, $0 \cdot 1 \mathrm{~mol} / 1 \mathrm{NaCl}$, and $0 \cdot 1 \%$ sodium azide for more than 5 days at $4{ }^{\circ} \mathrm{C}$ for BSA coating to prevent non-specific binding. The final volume percentage of GS4B was $15 \%$. Since the protein yield was usually the lowest in GSThPRL1-199, the concentration of the other fusion proteins was adjusted to that of GST-hPRL1-199 by BSA-coated GS4B (15\%) without fusion proteins based on the density of each band measured by National Institute of Health image.

\section{Binding study}

The scheme of the binding study is shown in Fig. 2a. The GST fusion proteins immobilized to GS4B $(100 \mu \mathrm{l})$ were incubated with $10 \mu \mathrm{l}$ serum in $200 \mu \mathrm{l}$ of reaction buffer $(0 \cdot 01 \mathrm{~mol} / 1$ phosphate buffer, $\mathrm{pH} 7 \cdot 0$, containing $0 \cdot 4 \mathrm{~mol} / 1$ $\mathrm{NaCl}$ and $0 \cdot 1 \%$ BSA) in a glass test tube $(1 \cdot 3 \times 10 \mathrm{~cm})$ with vigorous shaking at $37^{\circ} \mathrm{C}$ for $3 \mathrm{~h}$. After transferring the reaction mixture to a $1.5 \mathrm{ml}$ polypropylene tube, anti-PRL autoantibody-bound GS4B was centrifuged at 14000 r.p.m. for $5 \mathrm{~s}$, washed four times with $300 \mu \mathrm{l}$ of reaction buffer carefully not to aspirate the GS4B, and reconstituted with $200 \mu \mathrm{l}$ of the reaction buffer. The sera were similarly treated using GST alone-bound GS4B as a solid phase to determine the non-specific binding.

\section{ELISA for human immunoglobulin G (IgG)}

The concentrations of IgG attached to GST-hPRL fusion proteins were measured using a commercially available kit (Betyl Laboratories, Inc., Montgomery, TX, USA) according to the instructions with slight modification as shown in Fig. $2 \mathrm{~b}$. In brief, a 96-well uncoated microplate (Nunc, Roskilde, Denmark) was coated with anti-human IgG antibody for $4 \mathrm{~h}$ and washed three times with washing buffer $(50 \mathrm{mmol} / 1$ Tris$\mathrm{HCl}, \mathrm{pH} 8 \cdot 0$, containing $0 \cdot 14 \mathrm{~mol} / 1$ of $\mathrm{NaCl}$ and $0 \cdot 05 \%$ Tween 20), followed by incubation with $50 \mathrm{mmol} / 1$ Tris- $\mathrm{HCl}$ buffer, $\mathrm{pH} 8 \cdot 0$, containing $0 \cdot 14 \mathrm{~mol} / 1 \mathrm{NaCl}$ and $1 \% \mathrm{BSA}$ at $4{ }^{\circ} \mathrm{C}$ overnight for blocking. After washing the plate three times with washing buffer, $100 \mu$ of the post-reacted GS4B solution or human IgG standard were added in duplicate, and incubated at room temperature for $1 \mathrm{~h}$. After washing five times with $300 \mu \mathrm{l}$ of washing buffer, anti-human IgGhorseradish peroxidase conjugate was added followed by incubation for $1 \mathrm{~h}$. After washing five times with $300 \mu \mathrm{l}$ of the washing buffer, the peroxidase activity was measured using tetramethylbenzidine as a substrate (KPL, Gaithersburg, MD, USA). The absorbance was measured at $450 \mathrm{~nm}$ using a microtiter plate reader (Perkin-Elmer, Wellesley, MA, USA). The amounts of IgG specific for hPRL and its fragments were calculated by subtracting the binding to GST alone (nonspecific) from that to hPRL and its fragments. The binding of IgG was considered significant if more than $6.4 \mathrm{pg} / 1$ of IgG $($ mean $+2 \cdot 5$ S.D. in controls) was bound to solid phase. The intra- and interassay coefficients of variation were 4 and $14 \%$ respectively.

(a)

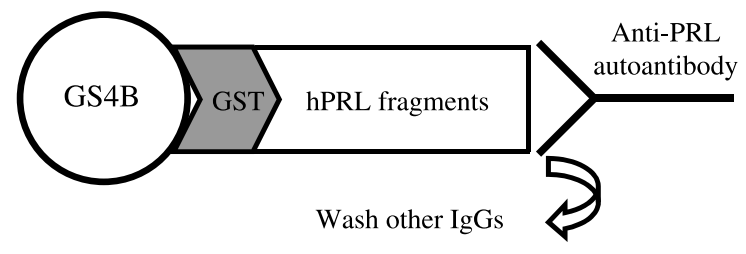

(b)
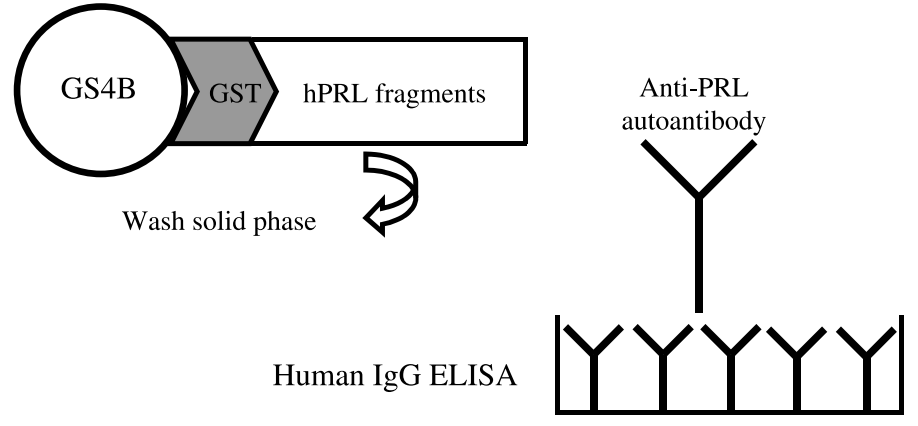

Rabbit anti-hIgG Ab

Figure 2 Schematic representation of a binding assay system to examine the binding of anti-PRL autoantibodies to hPRL and deletion mutants. (a) Immobilized GST-hPRL fusion proteins were incubated with sera and washed four times. (b) The autoantibody-bound fusion proteins were incubated with immobilized anti-human IgG antibody and the concentrations of the bound autoantibodies were measured using ELISA. 


\section{ELISA for $h P R L$}

hPRL concentrations were measured in duplicate using ELISA, which was previously developed by us (Hattori \& Inagaki 1997). Serum samples were diluted 20 -fold with $0 \cdot 01 \mathrm{~mol} / \mathrm{l}$ phosphate buffer, $\mathrm{pH} 7 \cdot 0$, containing $0 \cdot 4 \mathrm{~mol} / 1 \mathrm{NaCl}$ and $0 \cdot 1 \% \mathrm{BSA}$, and aliquots of the samples were treated with $12 \cdot 5 \%$ PEG for the evaluation of macroprolactinemia. The minimal detectable quantity was $0 \cdot 05 \mu \mathrm{g} / 1$ and the intra- and interassay coefficients of variation were 5 and $7 \%$ respectively. hPRL standard was based on WHO thirdinternational standard. Total and free hPRLs in 42 normal women were $12 \cdot 9 \pm 5 \cdot 7$, and $12 \cdot 3 \pm$ $5.9 \mu \mathrm{g} / 1$ respectively. The cut-off value for the diagnosis of macroprolactinemia was set as less than $40 \%$ recovery of $h P R L$ in the supernatant after treatment with PEG (Leslie et al. 2001). The autoantibody was found not to interfere with this assay system (Hattori et al. 1994).

All values were expressed as the mean \pm S.E.M. and statistical analysis was performed using unpaired Student's $t$-test. Differences were considered significant at $P<0 \cdot 05$.

\section{Results}

Using the developed autoantibody-binding assay system, we found that $\operatorname{IgG}$ was bound to full-length hPRL1-199 in significantly greater amounts in sera from 14 of 18 patients with macroprolactinemia $(19 \cdot 4 \pm 1 \cdot 6 \mathrm{pg} / \mathrm{l})$ than in controls $(1.9 \pm 0.4 \mathrm{pg} / \mathrm{l})$. However, four patients with macroprolactinemia (cases 15-18 in Table 1) did not show a significant increase, indicating that these patients do not possess anti-PRL autoantibodies despite being diagnosed with macroprolactinemia based on the PEG method.

To examine whether this system can detect specific binding of anti-PRL autoantibodies to hPRL, we performed displacement experiments. If the binding was specific, large amounts of hPRL standard co-incubated in the binding reaction mixture, would displace anti-PRL autoantibodies from the solid phase, leading to a decrease in the amount of IgG bound to the solid phase (Fig. 2). hPRL, but not PRL of other species such as bovine, porcine, rat, or human $\mathrm{GH}$, dose-dependently displaced the binding (Fig. 3), suggesting that the binding is specific to hPRL.

We performed binding studies using the solid phase of hPRL deletion mutants as shown in Fig. 4. Deletion of 34 amino acid residues from $\mathrm{N}$ - or C-terminal significantly reduced binding from $19 \cdot 4 \pm 1 \cdot 6 \mathrm{pg} / 1$ (hPRL1-199) to $9 \cdot 2 \pm 1 \cdot 5 \mathrm{pg} / 1$ (hPRL35-199) or $3 \cdot 3 \pm 1 \cdot 0 \mathrm{pg} / 1$ (hPRL1$165)$ respectively although the amount of $\operatorname{IgG}$ bound was still significantly higher than the controls $(P=0 \cdot 02$ and $0 \cdot 005$ for hPRL35-199 and hPRL1-165 respectively). The hPRL fragment with both $\mathrm{N}$ - and C-terminal 34 amino acid residues deleted (hPRL35-165) no longer showed significant binding $(1 \cdot 4 \pm 1 \cdot 0 \mathrm{pg} / \mathrm{l})$. N- (hPRL1-81) or C-terminal (hPRL135-199) fragment alone showed partial but significant binding $(3 \cdot 6 \pm 0 \cdot 7 \mathrm{pg} / 1$ in N-terminal and $8 \cdot 8 \pm 1 \cdot 7 \mathrm{pg} / 1$ in C-terminal fragments) compared to the controls.

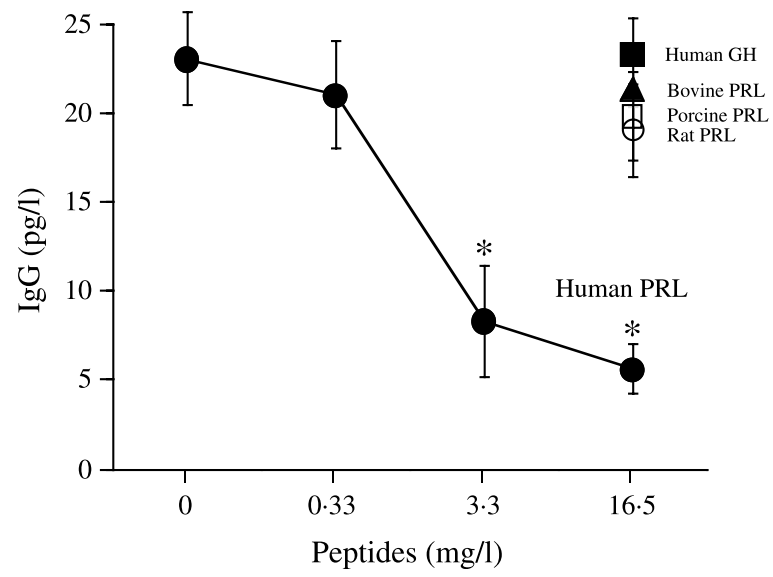

Figure 3 The displacement of anti-hPRL autoantibodies from immobilized full-length hPRL1-199 by human $(\mathbf{O})$, porcine $(\square)$, bovine $(\boldsymbol{\Delta})$, and rat PRL $(\mathrm{O})$ and $\mathrm{hGH}(\boldsymbol{\square}) .{ }^{*} P<0 \cdot 01$ as compared to bound IgG without the addition of $\mathrm{hPRL}$ standard. Bars indicate the mean \pm s.E.M. $n=3-4$.

\section{Discussion}

In this study, we established an anti-PRL autoantibody detection system to examine the epitopes on the hPRL molecule to the autoantibody. Since IgG is well known to adhere to the solid phase, it was critical to reduce non-specific binding. As shown in Fig. 2, we first incubated the serum with GST-hPRL fusion proteins immobilized on GS4B. During the reaction, not only free autoantibody but also autoantibody dissociated partially from endogenous PRL may bind to GST-hPRL fusion proteins. Because the affinity of anti-PRL autoantibodies is low compared to that of the reagent antibody (Hattori et al. 1992), it is probable that antihPRL autoantibody dissociates from GST-hPRL fusion proteins and binds to anti-human $\operatorname{IgG}$ antibody in human IgG ELISA. In addition to the separation of the solid phase, which directly contacts the serum, and the place for the final IgG determination, we used a high-salt buffer $(0.4 \mathrm{~mol} / \mathrm{l}$ $\mathrm{NaCl}$ ) during the reaction to prevent non-specific binding of IgG to the solid phase. hPRL has reportedly considerable homology with porcine, bovine, and rat PRL at $81 \cdot 4,73 \cdot 7$, and $61 \cdot 6 \%$ respectively (Sinha 1995). Therefore, it is surprising that hPRL, but neither PRL of other species nor hGH, dose-dependently displaced the binding of the autoantibody to solid-phase hPRL, indicating that the antibody is a hPRL-specific autoantibody. In addition, since we used recombinant hPRL as a solid-phase, anti-PRL autoantibody appears to recognize hPRL without modifications, which might break tolerance. Although careful handling of glutathione sepharose is needed, this method reduced the non-specific binding of $\operatorname{IgG}$ and enabled us to measure directly the amount of hPRL-specific autoantibody.

Sera from 14 of 18 patients with macroprolactinemia (78\%) showed significantly increased IgG binding to full-length hPRL1-199, suggesting that these patients had anti-PRL autoantibodies. The remaining four patients were 


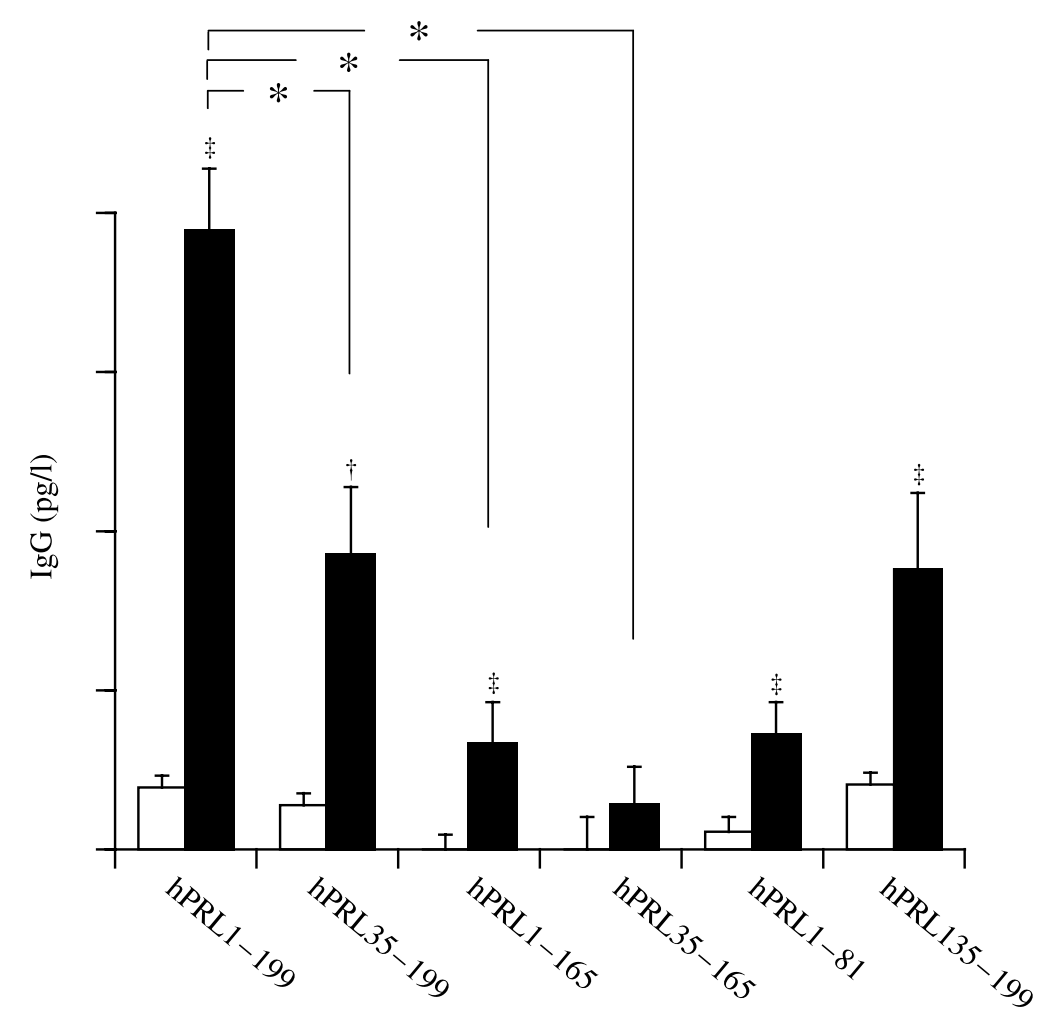

Figure 4 Binding of anti-PRL autoantibodies to full-length $\mathrm{hPRL}$ and its deletion mutants. Black bars represent sera from patients with anti-PRL autoantibodies $(n=14)$ and the white bars represent those from control hyperprolactinemic patients $(n=23) . * P<0 \cdot 01$ as compared to hPRL1-199. ${ }^{\dagger} P=0 \cdot 02,{ }^{\ddagger} P<0 \cdot 01$ as compared to each control (nonmacroprolactinemic hyperprolactinemia). Bars indicate the mean \pm s.E.M.

autoantibody-negative, although PEG treatment significantly precipitated PRL. These patients may have different types of association of PRL with IgG such as stable PRL-IgG complexes not displaceable by hPRL standard (Bonhoff et al. 1995, Walker et al. 1995). At present, macroprolactinemia is diagnosed using the PEG method, which is simple and correlates well to the results of the gel-filtration method (Vieira et al. 1998). However, $12 \cdot 5 \%$ PEG can precipitate not only IgG-bound PRL but also aggregate PRL or PRL-bound complexes to other large molecules (Ingham 1990). We previously reported a patient with macroprolactinemia who had a highly glycosylated PRL, but did not have anti-PRL autoantibodies (Hattori 1996). Therefore, macroprolactinemia is a heterogeneous entity, although many subjects have anti-PRL autoantibodies as shown in this study and other reports (Cavaco et al. 1995, De Schepper et al. 2003).

Since deletion of either $\mathrm{N}$ - or C-terminal 34 amino acid residues of PRL molecule markedly reduced the binding, major binding sites (epitopes) may be located in these residues. The sum of $\mathrm{IgG}$ bound to $\mathrm{N}$ - and C-terminal fragments did not reach that bound to full-length PRL, suggesting that anti-PRL autoantibodies recognize a conformational epitope that is lost when fragments of hPRL are used. The core hPRL structure is made up of four major $\boldsymbol{\alpha}$-helices (Teilum et al. 2005). The $\boldsymbol{\alpha}$-helix groups are in two anti-parallel pairs, helix 1 /helix 4 and helix 2 /helix 3 , each pair being packed more closely together. Therefore, several $\mathrm{N}$ - and C-terminal amino acid residues are located closely in the three-dimensional structure forming a part of binding site 1 to hPRL receptors. Thus, anti-PRL autoantibody and PRL receptors bind to the similar regions of PRL molecule, raising a possibility that the autoantibody may compete with the binding of PRL molecule to its receptors.

In this study, most patients with anti-PRL autoantibodies lacked clinical symptoms of hyperprolactinemia such as amenorrhea and galactorrhea, suggesting that the bioactivity of anti-PRL- $\operatorname{IgG}$ complex is lower than that of monomeric PRL. Four macroprolactinemic patients without anti-PRL autoantibody also lacked clinical symptoms. These patients had slight hyperprolactinemia and all the free PRL levels were normal. They might have other types of PRL-IgG complexes, which were reported as asymptomatic (Bonhoff et al. 1995). We previously demonstrated that the bioactivity of macroprolactin was almost equal to that of monomeric PRL using the $\mathrm{Nb} 2$ rat lymphoma cell line (Hattori \& Inagaki 1997) and reasoned that the minimum clinical symptoms by low accessibility to extravascular target cells due to their large molecular size. However, recently, using a homologous bioassay system (hPRL 
receptors), the bioactivity of macroprolactin has been reported to be lower than that of monomeric PRL (Glezer et al. 2006). It was claimed that a heterogeneous $\mathrm{Nb} 2$ rat lymphoma cell line is inappropriate for the evaluation of macroprolactin bioactivity. Although further study is necessary to better understand the biological features of anti-PRL autoantibodies, our data raise a possibility that anti-PRL autoantibodies may compete with the binding of the PRL molecule to its receptors, resulting in reduced bioactivity in vivo.

\section{Acknowledgements}

We thank Dr A F Parlow, a scientific director of the National Hormone and Pituitary Program, for supplying PRL and GH. We also thank Kansai Medical University Laboratory Center for providing experimental instruments and assistance. The authors declare that there is no conflict of interest that would prejudice the impartiality of this scientific work.

\section{References}

Bonhoff A, Vuille JC, Gomez F \& Gellersen B 1995 Identification of macroprolactin in a patient with asymptomatic hyperprolactinemia as a stable PRL-IgG complex. Experimental and Clinical Endocrinology and Diabetes 103 252-255.

Cavaco B, Leite V, Santos MA, Arranhado E \& Sobrinho LG 1995 Some forms of big big prolactin behave as a complex of monomeric prolactin with an immunoglobulin $\mathrm{G}$ in patients with macroprolactinemia or prolactinoma. Journal of Clinical Endocrinology and Metabolism 80 2342-2346.

De Schepper J, Schiettecatte J, Velkeniers B, Blumenfeld Z, Shteinberg M, Devroey P, Anckaert E, Smitz J, Verdood P, Hooghe R \& Hooghe-Peters E 2003 Clinical and biological characterization of macroprolactinemia with and without prolactin-IgG complexes. European Journal of Endocrinology 149 201-207.

Gibney J, Smith TP \& McKenna TJ 2005 The impact on clinical practice of routine screening for macroprolactin. Journal of Clinical Endocrinology and Metabolism 90 3927-3932.

Glezer A, Soares CR, Vieira JG, Giannella-Neto D, Ribela MT, Goffin V \& Bronstein MD 2006 Human macroprolactin displays low biological activity via its homologous receptor in a new sensitive bioassay. Journal of Clinical Endocrinology and Metabolism 91 1048-1055.

Hattori N 1996 The frequency of macroprolactinemia in pregnant women and the heterogeneity of its etiologies. Journal of Clinical Endocrinology and Metabolism 81 586-590.
Hattori N \& Inagaki C 1997 Anti-prolactin (PRL) autoantibodies cause asymptomatic hyperprolactinemia: bioassay and clearance studies of PRL-immunoglobulin G complex. Journal of Clinical Endocrinology and Metabolism 82 3107-3110.

Hattori N, Ishihara T, Ikekubo K, Moridera K, Hino M \& Kurahachi H 1992 Autoantibody to human prolactin in patients with idiopathic hyperprolactinemia. Journal of Clinical Endocrinology and Metabolism 75 1226-1229.

Hattori N, Ikekubo K, Ishihara T, Moridera K, Hino M \& Kurahachi H 1994 Effects of anti-prolactin autoantibodies on serum prolactin measurements. European Journal of Endocrinology 130 434-437.

Ingham KC 1990 Precipitation of proteins with polyethylene glycol. Methods in Enzymology 182 301-306.

Kleinberg DL, Noel GL \& Frantz AG 1977 Galactorrhea: a study of 235 cases, including 48 with pituitary tumors. New England Journal of Medicine 296 589-600.

Leslie H, Courtney CH, Bell PM, Hadden DR, McCance DR, Ellis PK, Sheridan B \& Atkinson AB 2001 Laboratory and clinical experience in 55 patients with macroprolactinemia identified by a simple polyethylene glycol precipitation method. Journal of Clinical Endocrinology and Metabolism 86 2743-2746.

Pascoe-Lira D, Duran-Reyes G, Contreras-Hernández I, Manuel-Apolinar L, Blanco-Favela F \& Leaños-Miranda A 2001 Frequency of macroprolactinemia due to autoantibodies against prolactin in pregnant women. Journal of Clinical Endocrinology and Metabolism 86 924-929.

Sinha YN 1995 Structural variants of prolactin: occurrence and physiological significance. Endocrine Reviews 16 354-369.

Sluijmer AV \& Lappöhn RE 1992 Clinical history and outcome of 59 patients with idiopathic hyperprolactinemia. Fertility and Sterility 58 72-77.

Teilum K, Hoch JC, Goffin V, Kinet S, Martial JA \& Kragelund BB 2005 Solution structure of human prolactin. Journal of Molecular Biology 351 810-823.

Vallette-Kasic S, Morange-Ramos I, Selim A, Gunz G, Morange S, Enjalbert A, Martin PM, Jaquet P \& Brue T 2002 Macroprolactinemia revisited: a study on 106 patients. Journal of Clinical Endocrinology and Metabolism 87 581-588.

Vieira JGH, Tachibana TT, Obara LH \& Maciel RMB 1998 Extensive experience and validation of polyethylene glycol precipitation as a screening method for macroprolactinemia. Clinical Chemistry 44 1758-1759.

Walker AM, Montgomery DW, Saraiya S, Ho TWC, Garewal HS, Wilson J \& Lorand L 1995 Prolactin-immunoglobulin G complexes from human serum act as costimulatory ligands causing proliferation of malignant $\mathrm{B}$ lymphocytes. PNAS 92 3278-3282.

Whittaker PG, Wilcox T \& Lind T 1981 Maintained fertility in a patient with hyperprolactinemia due to big, big prolactin. Journal of Clinical Endocrinology and Metabolism 53 863-866.

Received in final form 24 April 2006

Accepted 27 April 2006

Made available online as an Accepted Preprint 10 May 2006 